\title{
More care for patient's caregiver training: disregarded necessity besides pharmaceutical research
}

Volume 4 Issue I - 2015

\section{Opinion}

Researchers in pharmacy field have been dedicating their time and efforts in new drug discovery in order to find out sufficiently efficacious and safe treatment for different challenging diseases, either through investigations into medicinal plants like those of traditional Chinese medicine, Ayurveda, etc. or through chemical synthesis of new potential molecules. Besides working for achieving this goal; which is providing the patient with a recent drug that is effective and safe, we should think further and deeper about how to enable the patient to benefit from its maximum efficacy. Kinetic and pharmacodynamic studies aim to establish a precise profile of the medicine absorption, distribution, metabolism, and elimination on one side, and its mechanism of action in the other side, respectively. However, I suppose that it is of the same importance to ensure first that the medicine is administered by the patient and that the overall psychological and physical state of the patient is in favor of medicine efficacy. For this goal, I am writing this paper, where I put a spotlight on the importance of ensuring that the drug, which is the fruit of great efforts, time and money, can effectively fulfill its mission of treating the illness. In order to succeed in this mission, I recommend focusing more on the importance of caregivers, who are the nearest persons to the patient and a key element in the treatment.

Centuries of scientific research worldwide have lead to the discovery of numerous new drugs that have been commercialized to be prescribed properly by qualified doctors cooperating with pharmacists, and finally administered by the patients. These efforts are crucial and valuable; nonetheless, they could lead to no efficacy if the drug is not administered properly by the patient. The person suffering from an ailment or a very serious disease may need help from others in most cases. Some diseases may only slightly influence the patient's psychological state since the patient might feel that their body is not working well, and that they cannot practice and enjoy all the exercises and daily habits during the period of the disease. Other illnesses could be dramatically serious, where the patient may lose more vital abilities such as suffering from dementia and memory loss in the case of Alzheimer's disease, ${ }^{1}$ or having several health problems resulting from their main disease, such as the case of diabetic patients. ${ }^{2,3}$ Certainly, the patient is mostly in a pressing need of a caregiver.

The role of the caregiver is pivotal, and worth being taken considerably into consideration. The scientific research laboratories and the pharmaceutical companies are mostly focusing on the drug pharmacological and toxicological properties, and on the disease pathophysiological study. However, less investigation and interest is provided to the training of the patient's caregiver, whose presence and help are pivotal in medical treatment success. Obviously, the medicine should be administered on precise times and in the appropriate way to be efficacious and safe. While the patient, particularly if too young, too old or suffering from serious illnesses that affects their memory

\author{
Besma Boubertakh ${ }^{1,2}$ \\ 'Key Laboratory of Animal Models and Human Disease \\ Mechanisms of Chinese Academy of Sciences \& YunnanProvince, \\ Kunming Institute of Zoology, Kunming, Yunnan 650223, PR \\ China \\ ${ }^{2}$ University of Chinese Academy of Sciences, Beijing 10049, PR \\ China
}

Correspondence: Besma Boubertakh, Key Laboratory of Animal Models and Human Disease Mechanisms of Chinese Academy of Sciences \& Yunnan Province, Kunming Institute of Zoology, Kunming, Yunnan 650223, China, Emailwww.boubertakhbesma@outlook.com

Received: October 16, 2015 | Published: October 17, 2015

for instance, are unable to take their cure properly themselves, and, particularly in these cases, the presence of a well-formed caregiver is of vital importance. Herein, I propose that the caregiver should be cared for, and receive well training in order to ensure that they could deal appropriately with the patient. To achieve this noble endeavor, I suggest certain ways in the following passage.

The caregiver for an infant, an old person, or a patient suffering from a disabling disease, should continuously receive proper instructions. I suggest that the commercialized medicines should further include a caregiver instruction leaflet in addition to the usual patient information leaflet. This suggested paper, would include necessary information for the caregiver such as, remarks and notes about what they should pay attention to in order to ensure the well-being of the patient. For instance, this leaflet, could include instructions like how to deal with the symptoms, how to prevent disease aggravation, how to deal with possible crises, etc. It could mention also, some general suggestions and precautions about appropriate food diet, appropriate type of clothing and house furniture and environment arrangement suggestions for patients of special needs, such as those presenting a great risk of falling down because of their disease. Certainly, this could be specified for each patient via the doctor prescribing the medicine and the pharmacist according to the special requirements and circumstances of the disease. Herein, a discussion between the doctor or pharmacist and the caregiver, is also of considerable necessity via allowing them to follow and monitor the patient's treatment environment and to ensure that the medicine is taken under favorable physical and psychological conditions. The patient needs also continuous encouragement from their environment besides their medicine to achieve an optimal cure. Sensitization about the importance of the caregiver role during the medical treatment through media and scientific publication is also highly required via investigating their role and providing them with useful recommendations besides providing information about the disease and the medicine properties.

In the twenty first century, with the high prevalence of many 
diseases, almost everyone is either a patient or a caregiver, hence the importance of the education of the whole population about how to properly take care of a patient, through more frequent doctor/ pharmacist-caregiver communication, specialized leaflets in medicine package, inviting caregivers to attend medicinal conferences and meetings, dedicating the media besides scientific publications to further discuss and optimize the recommendations for the patient's caregiver to achieve a successful treatment. I insist on the importance of the caregiver training and education, and that it should not be overlooked anymore because they play a great role in the patient's treatment besides the doctor, the pharmacist and the scientific researcher; starting from encouraging the patient to drink more water and have a healthier diet and daily habits to ensuring consulting the doctor and administering the medicine following the appropriate schedule. The scientific research and the pharmaceutical production and commercialization need to focus and not neglect anymore the importance of the caregiver in order to achieve their goal of providing a better health state for the patient. Encouraging and educating the caregiver about the patient's disease requirements could undoubtedly help considerably in the promotion of humanity health.

\section{Acknowledgments}

None.

\section{Conflicts of interest}

Author declares there are no conflicts of interest.

\section{Funding}

None. 